\title{
Cross Talk Analysis on Multiple Coupled Transmission Lines; (The calculation of transfer functions on multiple coupled tansmission lines in an inhomogeneous dielectric medium)
}

\section{Dalby, Arne Brejning}

\section{Published in:}

Proceedings of the 24th European Microwave Conference

Link to article, DOI:

10.1109/EUMA.1994.337388

Publication date:

1994

Document Version

Publisher's PDF, also known as Version of record

Link back to DTU Orbit

Citation (APA):

Dalby, A. B. (1994). Cross Talk Analysis on Multiple Coupled Transmission Lines; (The calculation of transfer functions on multiple coupled tansmission lines in an inhomogeneous dielectric medium). In Proceedings of the 24th European Microwave Conference (Vol. Volume 2, pp. 1265-1270). IEEE. https://doi.org/10.1109/EUMA.1994.337388

\section{General rights}

Copyright and moral rights for the publications made accessible in the public portal are retained by the authors and/or other copyright owners and it is a condition of accessing publications that users recognise and abide by the legal requirements associated with these rights.

- Users may download and print one copy of any publication from the public portal for the purpose of private study or research.

- You may not further distribute the material or use it for any profit-making activity or commercial gain

- You may freely distribute the URL identifying the publication in the public portal 


\title{
Cross Talk Analysis on Multiple Coupled Transmission Lines.
}

(The calculation of transfer functions on multiple coupled transmission lines in an inhomogeneous dielectric medium)

Arne B. Dalby

Engineering Academy of Denmark. Dep. of Electronics, Building 451, DK 2800 Lyngby

\begin{abstract}
A flow graph relating voltages and the forward and reflected propagation modes $(\approx$ TEM) on multiple coupled transmission lines in an inhomogeneous dielectric medium is presented. This flow graph directly gives the different transfer functions, including S-parameters, in matrix form needed to calculate crosstalk on the lines. An 8 bit databus is analysed in the frequency- and time domain. This analysis shows, as expected, that crosstalk can be a problem in connection with high speed logic circuits.

The same databus is also analysed using the quasi-propagation-mode method proposed by Dalby [1-6]. This comparison shows, that the analysis and synthesis methods presented in [1-6] for interdigital filters to all practical purposes are accurate for frequencies below about 0.7 times the half-wave-length frequency for the lines.
\end{abstract}

\section{INTRODUCTION.}

Using the approach to the analysis of multiple coupled transmission lines presented in Dalby [5-7], a new way of illustrating the problem of time-domain- and frequency-domain analysis of cross-talk on $\mathrm{N}$ coupled transmission lines in an inhomogeneous dielectric medium is presented in the form of a matrix flow graph relating voltage vectors and incident- and reflected propagation modes (waves).

The flow graph representation makes it easy to analyse different cross-talk problems. The flow-graph takes into consideration the correct propagation modes ( $\approx$ TEM) on the $N$ coupled transmission lines and of course the terminating networks (impedance's).

It is also shown (proven) that it is not always necessary to know the correct propagation modes ( $\approx$ TEM) on the $N$ coupled transmission lines to find the frequency-response of the coupled transmission line system. The frequency, however, must be smaller than about 0.7 times the half-wave-length-frequency, If this is the case, it is possible to choose a set of propagation modes (quasi modes) almost arbitrarily. However, all the corresponding phase velocities must be taken into account, in principle $\mathrm{N} \cdot \mathrm{N}$ phase velocities instead of only $\mathrm{N}$.

Contrary to what one should think, because of the apparent added complexity, this freedom of choice of propagation modes is very advantageous in the synthesis and analysis of, e.g. interdigital filters, because among other things, the line lengths in interdigital filters are not identical, which means, that the flow graph method of analysis can not be used. The quasi mode method is described in [1-4].

\section{THE FLOW GRAPH.}

Figurel shows schematically a set of coupled transmission lines together with the definition of 3 voltage vectors. If the dielectric is homogeneous, the relations between the voltage vectors can be expressed in a way analogous to the relations between voltages on a single transmission line. If the dielectric is inhomogeneous, the relations are more complex as shown in figure 2.

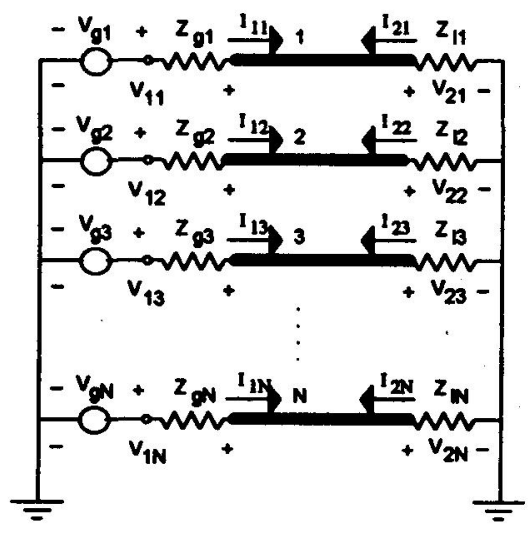

$$
v_{g}=\left\{\begin{array}{c}
v_{g 1} \\
\cdot \\
\cdot \\
v_{g N}
\end{array}\right\} \quad v_{1}=\left\{\begin{array}{c}
v_{11} \\
\cdot \\
\cdot \\
v_{1 N}
\end{array}\right\} \quad v_{2}=\left\{\begin{array}{c}
v_{21} \\
\cdot \\
\vdots \\
v_{2 N}
\end{array}\right\}
$$

Figure 1. A general coupled line circuit.

TEM mode propagation is assumed. This is permissible if the dimensions transversal to the transmission lines are small compared to the wave length, which in most cases will be true. The flow graph was developed from the theory of coupled transmission lines. The theory will not be presented here. The vectors $\mathbf{A}$ and $\mathbf{B}$ represent the amplitudes of the forward and backwards travelling propagation modes (voltages) respectively. There will be $\mathrm{N}$ possible propagation modes with different phase velocities.. 


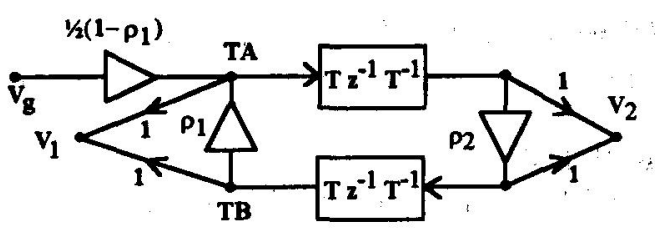

Figure 2. Flow graph showing the relation between voltage vectors.

A corresponding flow graph relating the current vectors $I_{1}$ and $I_{2}$, see figure 1, with $V_{\mathrm{g}}$ will look similar to the flow graph in figure 2. The matrices $\rho_{1}, \rho_{2}, T$ and $\mathrm{z}$ are explained below.

\section{TRANSFER FUNCTIONS.}

From the flow graph in figure 2, a number of transfer functions can be found. In (1) and (2) two of these are presented.

$$
\begin{aligned}
2 \cdot V_{2}= & \left(1+\rho_{2}\right) \cdot T \cdot z^{-1} \cdot T^{-1} \\
& \left(1-\rho_{1} \cdot T \cdot z^{-1} \cdot T^{-1} \cdot \rho_{2} \cdot T \cdot z^{-1} \cdot T^{-1}\right)^{-1} \cdot\left(1-\rho_{1}\right) \cdot V_{g} \\
2 \cdot V_{1}= & \left(1+T \cdot z^{-1} \cdot T^{-1} \cdot \rho_{2} \cdot T \cdot z^{-1} \cdot T^{-1}\right) \\
& \left(1-\rho_{1} \cdot T \cdot z^{-1} \cdot T^{-1} \cdot \rho_{2}-T \cdot z^{-1} \cdot T^{-1}\right)^{-1} \cdot\left(1-\rho_{1}\right) \cdot V_{g}
\end{aligned}
$$

The reflection coefficient matrices $\rho_{1}(3)$ and $\rho_{2}(4)$ are expressed in terms of the capacitance matrix $C$ for the $N$ coupled lines and the termination impedance matrices $\mathbf{Z}_{1}$ and $\mathbf{Z}_{2}(5)$. The impedance matrix $\mathbf{Z}_{1}$ must be diagonal, while $\mathbf{Z}_{2}$ need not be. Here both impedance matrices are diagonal. See figure 1 .

$$
\begin{aligned}
& \rho_{1}=\left(Z_{1} \cdot \mathbf{C} \cdot \mathbf{T} \cdot \mathbf{v} \cdot \mathbf{T}^{-1}+1\right)^{-1} \cdot\left(Z_{1} \cdot \mathbf{C} \cdot \mathbf{T} \cdot \mathbf{v} \cdot \mathbf{T}^{-1}-1\right) \\
& \rho_{2}=\left(Z_{2} \cdot C \cdot T \cdot v \cdot T^{-1}+1\right)^{-1} \cdot\left(Z_{2} \cdot C \cdot T \cdot v \cdot T^{-1}-1\right) \\
& \mathrm{z}_{1}=\left\{\mathrm{z}_{\mathrm{g} 1}, \mathrm{z}_{\mathrm{g} 2}, \cdots \cdots \mathrm{z}_{\mathrm{gN}}\right\}_{\text {diag. }} . \quad \mathrm{z}_{2}=\left\{\mathrm{z}_{\mathrm{g} 1}, \mathrm{z}_{\mathrm{g} 2}, \cdots \cdots \mathrm{z}_{\mathrm{gN}}\right\}_{\text {diag. }}
\end{aligned}
$$

$\mathbf{T}$ is an eigenvector matrix for $\mathbf{L} \cdot \mathbf{C}$, the product of the inductance matrix and the capacitance matrix for the coupled lines.

The matrices $\mathrm{v}$ and $\mathrm{z}$ are diagonal matrices where the diagonal elements are $\mathrm{v}_{\mathrm{p}}$, the propagation mode phase velocities, and $z_{p}=e^{s \cdot l / v_{p}}$ respectively. $z_{p}{ }^{-1}$ is the operator corresponding to the time delay $l / v_{p}$

$$
\mathbf{v}=\left\{\mathrm{v}_{1}, \mathrm{v}_{2}, \cdots \cdot \mathrm{v}_{\mathrm{N}}\right\}_{\text {diag. }}, \quad \mathrm{z}=\left\{\mathrm{z}_{1}, \mathrm{z}_{2}, \cdots \cdots \cdot \mathrm{z}_{\mathrm{N}}\right\}_{\text {diag. }}
$$

$v_{p}{ }^{-2}$ is an eigenvalue for the matrix $L \cdot C . l$ is the length of the transmission lines.

\subsection{Homogeneous dielectric.}

If the dielectric is homogeneous, and if there are no losses, there will be only one phase velocity $\mathbf{v}$. The matrix $\mathbf{T}$ can be chosen arbitrarily. The best choice is $T=1$, the unity matrix. This makes the transfer functions in (1) and (2) much simpler, and it is possible to make a sampled version of the time response for the system. The equations (in a loop) to be programmed for finding the voltage vector $V_{1}$, will be:

$$
\begin{aligned}
& n:=n+1 ; \quad B(n):=-\rho_{2} \cdot \mathbf{A}(n-1) ; \\
& \mathbf{A}(n):=\left(1-\rho_{111}\right) \cdot \mathbf{V}_{g}+\rho_{1} \cdot \mathbf{B}(n) ; \\
& \mathbf{V}_{1}:=\mathbf{A}(\mathbf{n})+\mathbf{B}(\mathbf{n}) ;
\end{aligned}
$$

Corresponding equations can be written for finding the vector $\mathbf{V}_{\mathbf{2}}$.

The sampling time is equal to the time delay $=\mathrm{T}=l / \mathrm{v}$, where $\mathrm{v}$ is the propagation velocity on the coupled line system, and $l$ is the length of the lines. A simple computer program can give the sampled voltages for a given input $\mathrm{V}_{\mathrm{g}}$ at $\mathrm{e}, \mathrm{g}$. line 1 . 
If the coupled transmission lines constitute e.g. a "data bus" or a "flat cable", the vector $\mathbf{V}_{1}$, should ideally be zero, except for the element $V_{11}$, otherwise there is cross talk on the lines. This may not be a problem, however, unless the voltages pass the threshold corresponding to logical "1".

If there are losses on the lines, these can easily be taken into account, approximately, by simply attenuating the "waves" (vectors) A and B in each loop of the program (equations (7) to (9)).

\subsection{Inhomogeneous dielectric.}

Normally the dielectric around the coupled lines is inhomogeneous, which means, that a time domain analysis is not possible, or at least only approximately.

In this case it is necessary to first calculate the transfer function in the frequency domain from the excitation to the point (transmission line end) where the cross talk analysis is wanted.

The expressions (1) and (2) for the transfer functions from the generator to the various line ends are, as it can be seen, rather complicated, but the transfer functions are relatively easy to calculate point by point on the frequency axis, using a computer program with routines for the multiplication of the complex matrices in (1) - (6) and of course a routine to find eigenvalues for the matrix $L \cdot C$.

A PASCAL program was made, which calculates the transfer functions in (1) and (2). They were found to be correct as indicated by the analysis of a number of interdigital filters designed by [1-4]. Losses have not been taken into account, but the method presented here can be extended to include losses.

A time domain analysis can be obtained from the transfer functions (1) and (2) by using the inverse FFT or by using the inverse Fourier transform.

By calculating the step response (multiplying the transfer function by $l / j \omega$ in the frequency domain), the time response corresponding to a pulse excitation can easily be found by simply subtracting a delayed version of the step response.

\section{CROSS TAlK ON A DATABUS.}

The general coupled line circuit in figure 2 also illustrates a typical databus.

In the following example an 8 strip databus realised on a printed circuit board as 8 coupled micro strips is assumed. The strips all have the same widths, and the spacing's are identical. The example may be a little extreme, because the spacings have been chosen relatively small, and no losses have been assumed. This was done to better illustrate the problems with cross talk. The values of terminating impedance's were chosen more or less arbitrarily in a range more or less typical for digital circuits. In this example they have been chosen to be real. In a more realistic example, capacitance's should be included, making the impedance's complex.

The physical dimensions, the properties of the dielectric substrate and the impedance values are given in table 1 . The The quasi mode parameters used to calculate the capacitance and inductance matrices for the coupled strips are presented an Appendix 1. The capacitance- and inductance matrices have been calculated using the methods and programs described in [1-4].

Table 1. Data for the databus: Substrate thickness $\mathrm{h}=0.0010 \mathrm{~m}$. All strip widths $=h$.

rel. dielectric constant $\varepsilon_{\mathrm{r}}=\mathbf{5 . 0 0}$. Spacings between the strips $=$ $0.2 \cdot h$.
Strip thickness $=0.050 \cdot \mathrm{h}$

Strip lengths $=100 \mathrm{~mm}$.

\subsection{Frequency response.}

Suppose the voltage on line 2 is wanted for the case of a step voltage excitation on line 1 . The transfer function from the generator at line 1 to the load at line 2 at the opposite end is shown in figure 3 for the frequency interval $0<f<4$ GHz. The frequency response shows a very confused picture. This is mostly due to the different propagation mode velocities, but also to the multiple couplings between the strips. However, depending on the physical dimensions (the substrate thickness) this response will be erroneous at higher frequencies because of higher order propagation modes and because of transverse propagation modes.

\subsection{Step- and pulse response:}

The step response can, as mentioned above, be found by taking the inverse Fourier transform (or the inverse FFT) of the transfer function multiplied by $1 / j \omega$. Input is at line 1 and output is at line 2 at the opposite end.

Figure 4 shows the step response as a function of time based on the frequency interval $0<f<12 \mathrm{GHz}$. The frequency step was $\Delta f=20 \mathrm{MHz}$., which means that the time response will be periodic with a period $=1 / \Delta \mathrm{f}=50 \mathrm{nsec}$, a period much larger than the time interval of interest. 


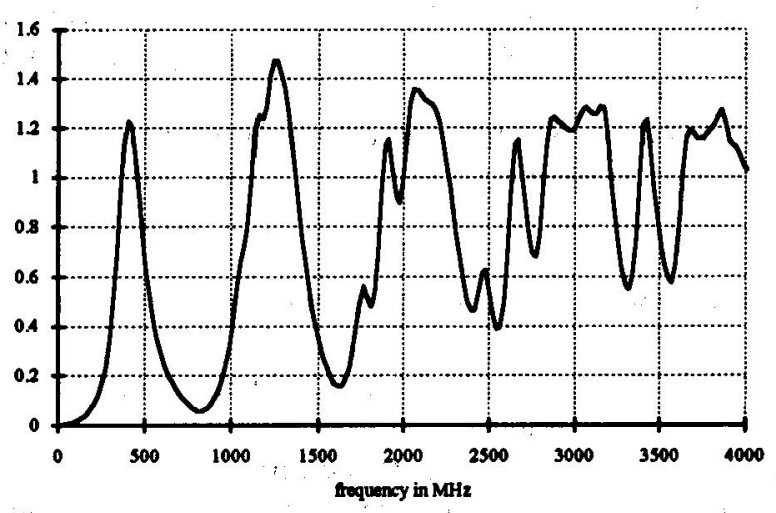

Figure 3. The frequency response for $0<\mathrm{f}<4 \mathrm{GHz}$.

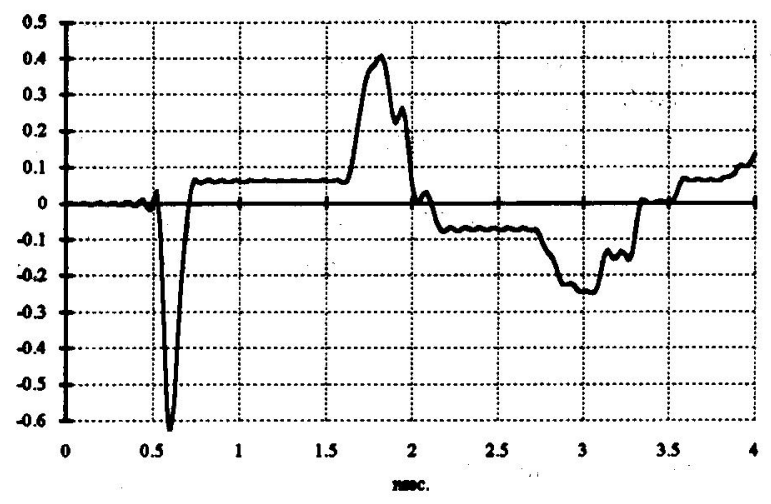

Figure 4. The step response based on the frequency response for $0<\mathrm{f}<12 \mathrm{GHz}$.

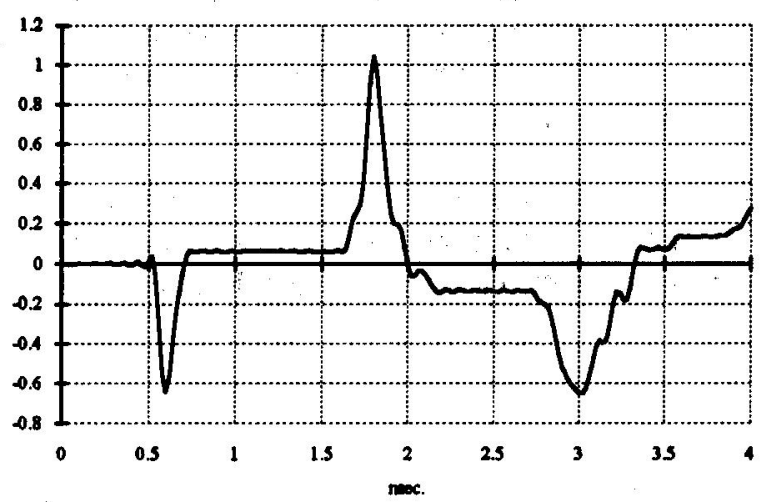

Fig. 5. The pulse response. Amplitude $=1$. Pulse width $=1.2 \mathrm{nsec}$.

Figure 5 shows the pulse response, assuming a pulse with a pulse width $=1.2 \mathrm{nsec}$. The response was calculated by subtracting a delayed step response from the step response in figure 4.

The time response in figure 5 shows, that a false signal may be detected on line 2 if very high speed circuitry is used. The example presented here may be a little extreme, but it does show, that there may be problems with cross talk on a databus.

\section{SCATTERING-PARAMETER-MATRICES.}

The scattering parameters can easily be expressed from the flow graph and the equations in chapters 3 and 5 . If the reference admittance matrix is chosen to be equal to the characteristic admittance matrix $y_{0}(12)$ for the coupled line system, then the S-parameter matrices will be as shown in (10) and (11)

The matrix $y_{0}$ is not a simple diagonal matrix, and the elements are not identical to say $20 \mathrm{mS}$, so the equations (10) and (11) for the S-parameters are not easily employed. 


$$
\begin{aligned}
& S_{21}=S_{12}=T \cdot z^{-1} T^{-1} \\
& S_{11}=S_{22}=0 \\
& y_{0}=C \cdot T \cdot v \cdot T^{-1}
\end{aligned}
$$

If both the load- and generator impedance matrices $\mathrm{Z}_{1}$ and $\mathrm{Z}_{2}$ are identical and equal to a diagonal reference matrix $Z_{0}$ with elements equal to, e.g. $50 \Omega$, then the scattering parameter matrices will be:

$$
\begin{aligned}
& S_{11}=S_{22}=T \cdot z^{-1} \cdot T^{-1} \cdot \rho \cdot T \cdot z^{-1} \cdot T^{-1} \\
& S_{21}=S_{12}=(1+\rho) \cdot T \cdot z^{-1} \cdot T^{-1} \cdot\left(1-\rho \cdot T \cdot z^{-1} \cdot T^{-1} \cdot \rho \cdot T \cdot z^{-1} \cdot T^{-1}\right)^{-1} \cdot(1-\rho) \\
& \text { where } \\
& \rho=\left(Z_{0} \cdot C \cdot T \cdot V \cdot T^{-1}+1\right)^{-1} \cdot\left(Z_{0} \cdot C \cdot T \cdot M \cdot T^{-1}-1\right)
\end{aligned}
$$

\section{QUASI MODE ANALYSIS:}

The frequency response in figure 3 could also have been calculated using the method described in [1-4], that is, using quasi propagation modes instead of the "correct" propagation modes. The method, however, can only be used for frequencies below about 0.7 times the half wave frequency. For the 8 strip databus this frequency is about $550 \mathrm{MHz}$. In the quasi mode approach, the matrix $\mathbf{T}$ is substituted by a matrix $\mathbf{Q}$, which in principle could be chosen almost arbitrarily. A special choice is made, a choice which makes the calculation of $\mathbf{L}$ and $\mathbf{C}$ relatively easy using empirical formulas. The choice is also ideally suited for filter synthesis purposes. An apparent drawback to the method is that the matrices $\mathrm{v}$ and $\mathrm{z}$ will no longer be diagonal. The flow graph can in fact no longer be employed as it is. Instead a model based on admittance parameters is used. See [1-7] for a more thorough explanation.

\section{Frequency response $|\mathrm{V} 22 / \mathrm{Vg}| \mid$ for $0<\mathrm{f}<600 \mathrm{MHz}$}

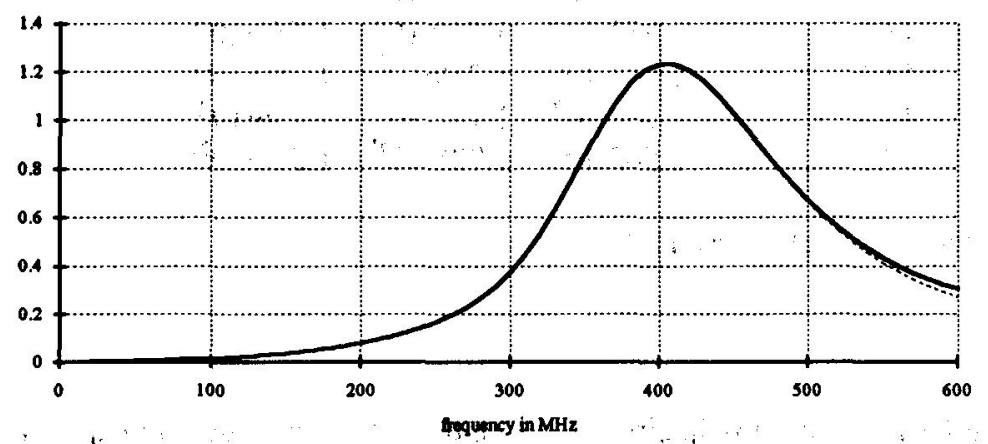

Figure 8. The frequency response calculated using the quasi mode approach. The dotted curve is the response calculated using the flow graph.

Figure 8 shows, that the frequency response calculated using the quasi mode approach and the one calculated using the flow graph are identical, to all practical purposes, up to about $550 \mathrm{MHz}$. The quasi mode parameters are presented in Appendix 1.

The quasi mode method, in which admittance parameters for the 2.8 port network are calculated using the quasi mode parameters in Appendix 1, will not be explained here. The reader is referred to [1-6].

\section{Why use the quasi mode approach?}

- The correct method based on the flow graph in figure 2 is only valid when the strip lengths are identical. For interdigital micro strip filters this is not the case. The strip lengths must be adjusted so that each strip approximately has a length equal to a quarter wave length at the filter centre frequency for the most important propagation mode or modes for the strip.

- The synthesis procedure for interdigital filters must necessarily be an iterative procedure, where it is important to have parameters which vary in a predictable way with physical dimensions. These parameters must also have a simple relationship to the filter constants found in handbooks for, e.g. chebyshev normalised filters. This is true for the quasi mode approach.

- In the design procedure for interdigital filters, it is easy to find the optimum length for each of the micro strips if 
the quasi mode method is used.

- The quasi mode design method takes losses into account based on empirical formulas. The method is employed in a PASCAL program, which gives the physical dimensions for the desired filter and predicts the insertion loss within about $1 \mathrm{~dB}$ 's accuracy! Work and money can thus be saved, because it is easily shown, whether a certain filter is realisable or not.

- It is of course possible to use a "more correct" method in the design and analysis of interdigital micro strip filters, but the work involved would be rather horrendous. This may be the reason so very few articles exist on the design of interdigital micro strip filters.

- The quasi mode method has proven its value in the design of a number of filters which all met the desired specifications [1-7].

- The quasi mode approach also makes it fairly easy to construct empirical formulas for finding the capacitanceand inductance matrices for the coupled lines. [1-3]

\section{CONCLUSION.}

It has been shown that cross talk analysis on multiple coupled transmission lines in an inhomogeneous dielectric medium (a databus) is fairly easy using the flow graph in figure 2 for the transmission modes.

It has also been shown in this paper, through a practical example, that the quasi transmission mode approach is accurate to all practical purposes for frequencies below about 0.7 times the half wave frequency (the frequency where the line lengths correspond to $\mathrm{ca}$. a half wave length). The advantages of the quasi mode analysis and synthesis methods compared to the more correct ones based on the flow graph have also been explained.

\section{APPENDIX 1.}

Quasi mode parameters:

$\begin{array}{ccccccc}\text { strip } & 1 / y_{\text {even }} & 1 / y_{\text {odd,left }} & 1 / y_{\text {odd, right }} & v_{\text {even }} & v_{\text {odd,left }} & v_{\text {odd,right }} \\ 1 & 87.4 & 87.3 & 37.9 & 1.545 \cdot 10^{8} & 1.546 \cdot 10^{8} & 1.738 \cdot 10^{8} \\ 2 & 141.4 & 45.1 & 43.6 & 1.368 \cdot 10^{8} & 1.756 \cdot 10^{8} & 1.757 \cdot 10^{8} \\ 3 & 145.5 & 44.1 & 44.1 & 1.363 \cdot 10^{8} & 1.759 \cdot 10^{8} & 1.759 \cdot 10^{8} \\ 4 & 145.5 & 44.1 & 44.1 & 1.363 \cdot 10^{8} & 1.759 \cdot 10^{8} & 1.759 \cdot 10^{8} \\ 5 & 145.5 & 44.1 & 44.1 & 1.363 \cdot 10^{8} & 1.759 \cdot 10^{8} & 1.759 \cdot 10^{8} \\ 6 & 145.5 & 44.1 & 44.1 & 1.363 \cdot 10^{8} & 1.759 \cdot 10^{8} & 1.759 \cdot 10^{8} \\ 7 & 141.4 & 43.6 & 45.1 & 1.368 \cdot 10^{8} & 1.757 \cdot 10^{8} & 1.756 \cdot 10^{8} \\ 8 & 87.4 & 37.9 & 87.3 & 1.545 \cdot 10^{8} & 1.738 \cdot 10^{8} & 1.546 \cdot 10^{8}\end{array}$

For an explanation of the quasi mode parameters, see [1-4].

\section{REFERENCES}

1. A. B. Dalby. Analysis and Synthesis of Micro strip circuits with more than two Coupled Strips. 7th European Microwave Conference. Book of proceedings pp 441-445. September 1977.

2. A. B. Dalby. Interdigital Micro strip Circuit Parameters using Empirical Formulas and Simplified Model. IEEE MTT-S International Microwave Symposium Digest pp 223-226. June 1979.

3. A. B. Dalby. Interdigital Micro strip Circuit Parameters using Empirical Formulas and Simplified Model. IEEE Transactions on MTT, vol. MTT-27. No. 8, pp 744-752. August 1979.

4. A. B. Dalby. On Coupled Thick Micro strips. 16th European Microwave Conference in Dublin, September 8-11,1986. Conference proceedings page 535-540. (A revised copy can be obtained from the author)

5. A. B. Dalby. A Microwave Digital Filter or a Digital Microwave Filter? Onzieme Colloque Gretsi - Nice du ler au 5 Juin 1987. Book of proceedings pp 25-28.

6. A. B. Dalby. From Microwave Filter to Digital Filter and Back Again. 19th European Microwave Conference in London, September 4-7,1989. Conference proceedings pp 892-897.

7. A. B. Dalby. A State Space Wave Digital Filter.10th European Conference on Circuit Theory and Design in Copenhagen, September 1991. Book of proceedings pp 187-196. 